\title{
Gathering and Conceptualizing Plan-Based Robot Activity Experiences
}

\author{
Vahid Mokhtari, Gi Hyun Lim, Luís Seabra Lopes \\ and Armando J. Pinho
}

\begin{abstract}
Learning from experiences is an effective approach to enhance robot's competence. This paper focuses on developing capabilities for a robot to obtain robot activity experiences and conceptualize the experiences as plan schemata, which are used as heuristics for the robot to make plans in similar situations. The plan-based robot activity experiences are obtained through human-robot interactions where a teaching action from a command-line user interface triggers recording of an experience. To represent human-robot interaction activities, ontologies for experiences and user instructions are integrated into a robot ontology. Recorded experiences are episodic descriptions of the robot's activities including relevant perceptions of the environment, the goals pursued, successes, and failures. Since the amount of experience data is large, a graph simplification algorithm based on ego networks is investigated to filter out irrelevant information in an experience. Finally, an approach to robot activity conceptualization based on deductive generalization and abstraction is presented. The proposed system was demonstrated in a scenario where a PR2 robot is taught how to "serve a coffee" to a guest, in the EU project RACE.
\end{abstract}

Keywords Plan-based robot activity $\cdot$ Experience gathering $\cdot$ Ego network $\cdot$ Plan schema $\cdot$ Abstraction and generalization $\cdot$ Conceptualization

\footnotetext{
V. Mokhtari ( $\varangle) \cdot$ G.H. Lim $\cdot$ L. Seabra Lopes · A.J. Pinho

IEETA, University of Aveiro, Aveiro, Portugal

e-mail: mokhtari.vahid@ua.pt

URL: http://wiki.ieeta.pt/

G.H. Lim

e-mail: lim@ua.pt

L. Seabra Lopes

e-mail: 1sl@ua.pt

A.J. Pinho

e-mail: ap@ua.pt

L. Seabra Lopes · A.J. Pinho

DETI, University of Aveiro, Aveiro, Portugal 


\section{Introduction}

Service robots are expected to increasingly interact and collaborate with humans. These robots have to autonomously adapt their behaviors and actions to unforeseen situations. Therefore, learning is one of the most important properties of service robots, since it enables robots to learn new skills and acquire and adapt knowledge of the system to new contexts and situations. In this concern, the RACE (Robustness by Autonomous Competence Enhancement $)^{1}$ project has focused on developing a framework to learn from experiences. Robot's competence is obtained by abstracting and generalizing from experiences, thus broadening task planning and behavior adaptation. The principal objective of RACE is to build an artificial cognitive system, which enables a service robot to make a high-level understanding of the world by storing and exploiting appropriate memories of its experiences. These experiences are records of past happenings stored by the robot which are interpreted according to the robot's conceptual framework, in a coherent subset of space-time.

This paper presents our latest achievements in obtaining plan-based robot activity experiences with emphasis on filtering out irrelevant information, and conceptualizing the experiences as plan schemata. We describe the research approach and the infrastructure designed for supervised experience gathering of plan-based robot activities. For this purpose, ontological concept representations and a command-line interface have been developed to support human-robot interaction, allowing an inexpert user to instruct a robot to perform tasks as well as teach new task concepts. The teaching action from the user triggers off gathering and filtering of the experiences relevant for conceptualizing the new task. A conceptualization method based on deductive generalization and abstraction is then proposed.

This paper is organized as follows: Sect. 2 is dedicated to review the related literature addressing experience gathering and task learning from interaction. The overall RACE architecture and the proposed ontology of experience and humanrobot interaction are presented in Sects. 3 and 4 respectively. Section 5 represents the user interface for human-robot interaction. Moreover, temporal segmentation heuristics for experience extraction and proposed filtering approach are explained in this section. Section 6 is devoted to describe the conceptualization approach, and finally conclusion is represented.

\section{Related Work}

Different approaches to acquire experiences and learn high-level action models from environment have been explored, ranging from unsupervised to supervised experience acquisition [1-4]. In this particular context, human-robot interactions help to

\footnotetext{
${ }^{1}$ RACE is funded by the EU Seventh Framework Program theme FP7-ICT-2011-7, grant agreement No. 287752.
} 
endow robots with the capabilities to learn easily how to perform and generalize a task by observing several demonstrations.

Kirsch [5] addressed the integration of programming and learning in a control language for autonomous robots. The proposed robot learning language (RoLL) is built on the concept of experiences and supports a semi-supervised approach to experience gathering. An experience is a summary of the internal and external state changes during an episode. The author distinguished between directly observed experiences raw experiences and transformed experiences abstract experiences suited for learning. Since all experiences are not needed permanently, permanent and transient experiences are addressed. Permanent experiences are stored in files or a database, and transient ones exist until their data is passed on to the next experience processing step.

Pardowitz et al. [4] presented an incremental task knowledge learning by demonstration which benefits from speech comments to gains more knowledge when more task demonstrations become available. The robot starts with a formal definition of the structure of a task, which allow for multiple orderings of the primitive operations. Then, at each human demonstration of the task, the robot acquires knowledge about the best sequences. This involves segmenting the demonstration into basic actions and mapping the segmented sequence to the given task model. This method is based on extracting and comparing features of subtasks. The acquired knowledge is incrementally incorporated into the task model. Relevance of task features (such as manipulated objects or action pre-/post-conditions) is estimated based on vocal comments provided by the user during demonstrations. A set of feature weights is updated at each demonstration of the same task.

In another work toward learning from demonstration, Ekvall and Kragic [6, 7] presented a task-level planning system. Three leaning approaches have been investigated in their papers: imitation learning to represent task learning, learning from human explanation and generalizing from multiple observations where a task is represented by its goal configuration and task constraints. The authors addressed the term of task constraints to indicate that the robot has to avoid solving a task in a wrong way. Constraints are identified either using the teacher instructions or generalizing from multiple observations. Constraints are then considered in the planning to choose the best strategy.

Nicolescu and Mataric [8] presented an approach for teaching by demonstration to enable a robot to learn and refine representations of complex tasks. The authors assumed an equipped robot with a set of skills and then focused on high-level task learning from the existing skills. Simple informative cues (i.e., take, drop etc.) in the form of verbal instructions are used by a teacher to enable the robot to distinguish between relevant and irrelevant information during the learning process. An abstract network behavior approach is proposed for learning task representation. To generalize several demonstrations of the same task into a single directed acyclic graph representation, a common sequence of behaviors is computed based on the longest list of common nodes between different topological representations of tasks. Finally, natural feedback cues (i.e., bad, come, and go) are provided by the teacher through speech to allow the robot to further refine this representation. 
Sehou et al. [9] presented an HRI system for industrial task-level-programming. They proposed a three-layered abstraction architecture of device primitives, skills, and tasks. In the lowest layer, device primitives are the basic functionalities and motions. The skill layer, in the middle, contains object-centered capabilities, i.e., "pick", "place", etc. And in the highest layer, tasks are created by sequencing parameterized skills. Learning a new task is carried out through specification and teaching phases. In the specification phase, a sequence of skills and initial parameters are chosen using a graphical interface, and during the teaching phase locational parameters are obtained through kinesthetic teaching.

\section{Architecture of RACE}

In the RACE architecture [10], an RDF database is used as Blackboard, which serves as a working memory for computations and communications of all modules, as shown in Fig. 1. Data stored in blackboard is mostly of semantic nature, for which an ontology has been developed. It keeps track of the evolution of both the internal state of the robot and the events observed in the environment. The blackboard can be read or written by different modules. Every unit of data written to the Blackboard is called fluent, which describes an instance of some concept in the ontology. Blackboard and semantic memory are interchangeably referred in this paper.

The Reasoning and Interpretation includes high-level interpreter, and temporal and spatial reasoners. Perception contains several modules for symbolic proprioception and exteroception which generate occurrences. New concepts are added to the Memory and stored in an OWL ontology format. User Interface sends instructions that generate a goal which is then relayed to Planning. Planning is carried out using JSHOP2, a Hierarchical Task Network (HTN) planner [11, 12]. The generated plan is dispatched to the Plan Execution and Management, and the robot is finally demanded to perform actions. The history of occurrences in the memory is

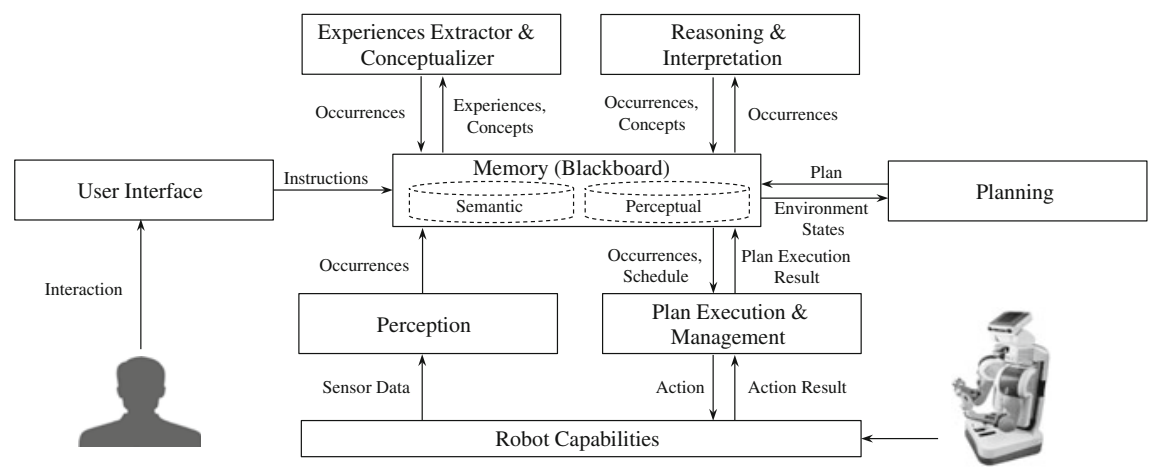

Fig. 1 The RACE architecture 
continuously observed by the Experience Extractor to detect and extract potentially relevant experiences, based on plan structure, user feedback, and similarity with stored experiences. Experiences are subsequently fed to Conceptualizer to generate and store new concepts or update existing concepts, resulting in more robust and flexible future plans. In this paper, User Interface, Experience Extractor and Conceptualizer are addressed.

\section{Ontological Aspects}

Many concepts relevant for planning and execution of robot tasks are covered in the RACE OWL2 ontology [10]. To support experience gathering, concepts to represent experiences and user instructions are also included in the ontology. This section elaborates on these ontological aspects.

\subsection{Ontology of Experiences}

Experiences are the main source of information used for learning how to achieve a more robust robot behavior. Thus in this project, "experiences" play a role similar to "cases" in Case-Based Reasoning, "episodes" in Cognitive Science or "instances" in Instance-Based Learning. They arise from robot observations and activities. We introduce a basic distinction between spatial and dynamic experiences. Spatial experiences can be related to the evolutionary roots of human semantic memory [13]. They are relevant for conceptualizing the spatial and relational structure of passive physical objects and scenes, in particular the shape and appearance of objects and the layout of scenes. By contrast, dynamic experiences contain data that describes activities as sets of occurrences. Considering the scope of this paper, the ontological concepts only related to dynamic experiences are represented in Listing 1.

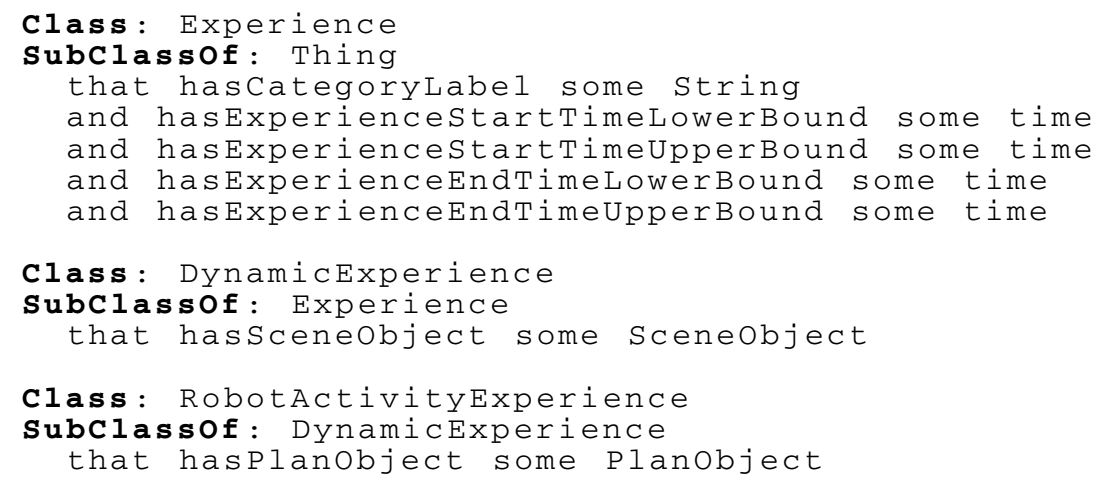

Listing 1 Ontology of experiences in OWL2 Manchester Syntax. 
Experience concept represents an experience record containing data that describes an instance of a category to be learned along with a name provided by an instructor or an unsupervised internal process (CategoryLabel). DynamicExperience is an experience relevant for conceptualizing activities in terms of sets of occurrences in a coherent subset of space-time. RobotActivityExperience is a dynamic experience containing data about an activity of the robot, extracted from the occurrence history during the execution of that activity. The experience data includes the underlying goals, executed plan, a set of occurrences, and success information. Robot activity experiences are relevant for conceptualizing a given category of robot activities.

\subsection{Human-Robot Interaction Ontology}

In RACE project, human-robot interaction is essentially used for supervised experience gathering. It will be used in situations where the robot fails to autonomously deal with exceptional situations as well as for teaching knowledge relevant for new tasks. The human-robot interaction ontology is centered on the instructor activities. Listing 2 shows part of the InstructorActivity ontology considering the scope of the paper.

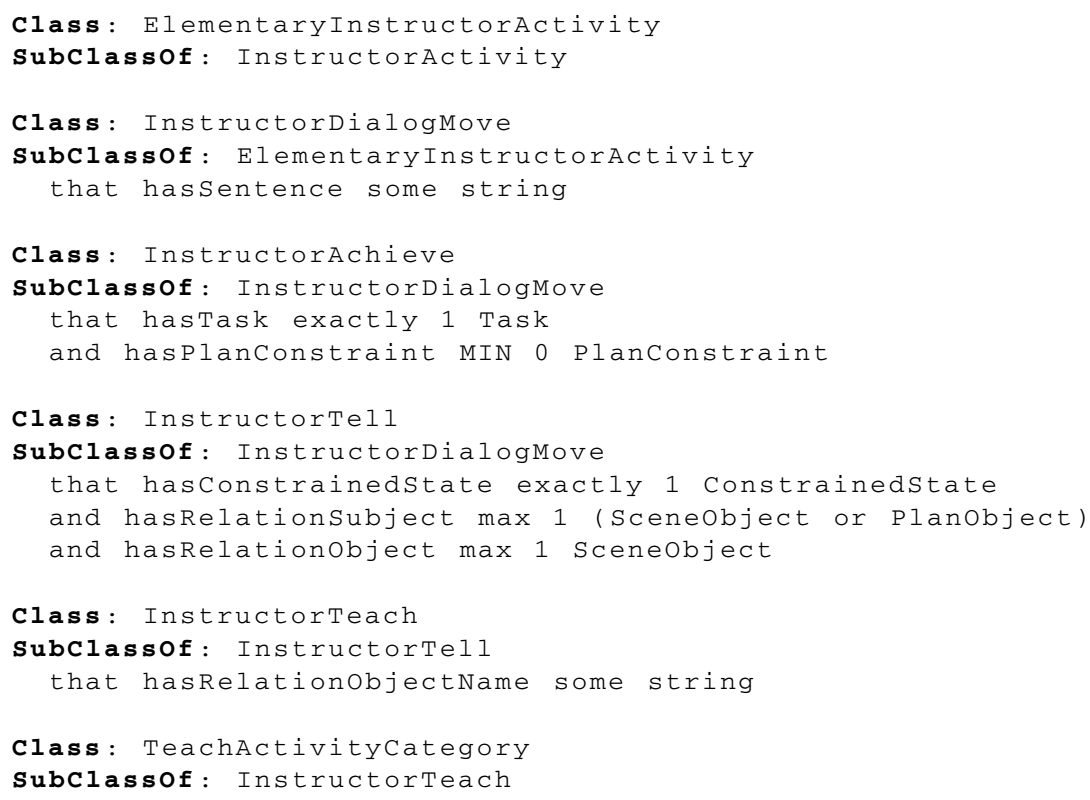

Listing 2 Ontology of instructors related to robot activities in OWL2 Manchester Syntax.

InstructorAchieve is a dialog move of imperative nature, i.e., it is used to request the robot to achieve some goal or to perform some activity. Instructor $\mathrm{Tel} 1$ is a dialog move of general-purpose declarative nature. InstructorTeach 
is used for providing information in terms of assertions about the scene. A special case is TeachActivityCategory which is useful for learning a new taught task.

\section{Recording Robot Activity Experiences}

During execution, each module adds fluents to represent the current state and to reflect its perception of environment into the blackboard. Robot activity experiences are acquired from semantic memory via a human-robot interaction interface, and are filtered to extract the most relevant knowledge.

\subsection{Interactive Teaching for Experience Acquisition}

A command-line interface has been developed to support communication between the robot and a human user. The interface allows the user to provide instructions for the successive steps in a task, such as driving to locations, picking, and placing objects. The user can also teach a set of performed activities with a given name. The following types of instructions are used for experience gathering:

- achieve this instruction corresponds to the InstructorAchieve concept and takes as input a task to be performed with respective arguments:

Format: achieve <task name> <task arguments>

- teach_task this instruction corresponds to the TeachActivityCategory concept and takes as input the name of the task and its arguments that user wishes to teach:

Format: teach_task <task name> <task arguments>

This interface is tightly integrated with the semantic memory. When the robot completes the task or achieves the goal, a teach_task instruction triggers the experience extraction. Temporal segmentation heuristics-e.g., the start time of the first achieve and the end time of the teach_task instructions-are taken into account to extract a subset of fluents related to the given task [14]. All fluents active (started and/or ended) within this interval become part of the experience pertaining to the new activity taught by the instructor [15]. Listing 3 shows an example to the sequence of instructions to teach the robot the standard method for serving coffee to guest.

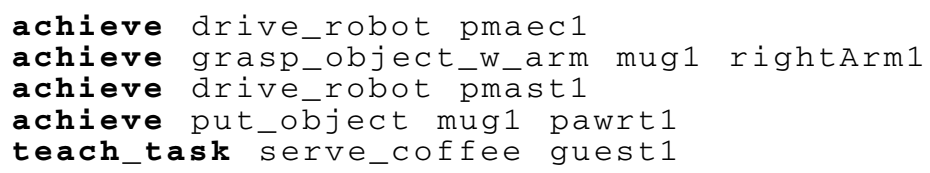

Listing 3 Sequence of instructions to teach the robot how to serve a coffee to guest1. 
Achieve instructions specify instances of the Task concept to be executed. The user interface creates a fluent for every achieve instruction which is dispatched to the JSHOP2 planner to generate a plan. The Planner decomposes the task into subtasks where operators are used to perform primitive tasks. Experience gathering is immediately carried out after teach_task instruction.

\subsection{Filtering Robot Activity Experience}

The semantic memory is taken into consideration as a graph, which has been frequently utilized to encapsulate the relationships between objects [16]. The graph is an ordered $G=[V, E]$, where $V$ is a set of vertices representing fluents in semantic memory, and $E$ is a set of links between fluents. Since the semantic memory contains insignificant and irrelevant fluents during task execution, a standard graph simplification approach based on ego network is employed to filter out such irrelevant fluents.

An ego network is a network centered on a specific focal node which is called the ego [17], and the nodes connected to the ego are called alters. A network may contain one or more egos. To construct an ego network, some nodes are selected as egos, and their alters are decided based on the length of the shortest path to an ego. The result is a subgraph describing the neighborhoods surrounding the egos, which may reveal something important from the egos' perspective. In the current state of implementation, a one-step neighborhood has been used, which includes alters that are directly adjacent to the ego. These networks are also known as neighborhood networks or first order neighborhoods of an ego node.

In RACE an HTN planner has been utilized to plan compound tasks [12]. HTN planning is carried out by decomposing non-primitive tasks recursively into subtasks to reach the leaves of task hierarchy. The leaf nodes contain primitive tasks which can be performed directly using the planning operators. Every operator is a triple, $o=[\operatorname{op}(\operatorname{args})$, precond, postcond $]$, where $\operatorname{op}(\operatorname{args})$ is the operator name with a list of its arguments, and precond and postcond are respectively sets of properties that should be true before and after applying the operator $o$.

Since the experience extraction is based on plan-based robot activities, the egos are simply defined according to the fluents involved in the plans' operators,

$$
\text { egos }=\{u \mid u \in(\text { args } \cup \text { precond } \cup \text { postcond })\},
$$

where args, precond and postcond are respectively the arguments, preconditions and postconditions of the applied operators during the execution of the plans. The alters are subsequently obtained by computing the first order neighborhoods for egos and recorded as parts of the experience,

$$
\text { alters }=\{v \in \text { fluents } \mid \forall u \in \operatorname{egos}(v \downarrow u)\} .
$$


To fully grasp the significance of filtering out irrelevant fluents, the Yifan $\mathrm{Hu}$ Multilevel layout graph visualization has been used, since it is optimized for the visualization of large graphs [18]. Figure 2 shows how the experience content is largely simplified graph after filtering.

\section{Conceptualizing Plan-Based Robot Activities}

Since experiences are extracted, robot utilizes its experiences to solve new problems based on analogies with previous cases or episodes stored in memory. One of the pursued approaches is based on abstracting and generalizing such experiences. The result of processing an experience is an activity schema (conceptualization) applicable to a wide range of problems. The main idea is extended from the works of Seabra Lopes [19] by enhancing the feature extraction module. A robot activity experience is represented as a triple,

$$
\text { [Task, KeyProps, SeqOp] }
$$

where,

- Task is the taught task name and its arguments as given by the teach_task instruction, e.g., serve_coffee (guest 1 );

- KeyProps is a subset of propositions, which are true in some stages during task execution, e.g., throughout (at (guest1, sawt1)), at_start (on (mug1, paelc1)), at_end (on (mug1, pawrt1));

- SeqOp is the applied sequence of primitive operators.

KeyProps contains three different types of propositions, at_start (true at initial state), throughout (always true during task execution) and at_end (true at final state).

The proposed conceptualization approach is described along with an example. For this purpose, the sequence of instructions to teach the standard method for serving coffee illustrated in Listing 3 are used (Sect. 5.1). Listing 4 shows the extracted experience represented as above triple. A total of 113 key propositions and 16 primitive operators were extracted from semantic memory after running serve a coffee scenario.

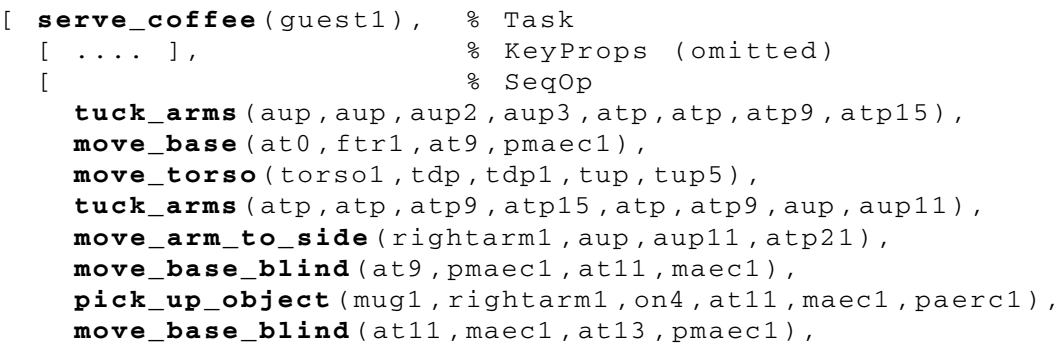



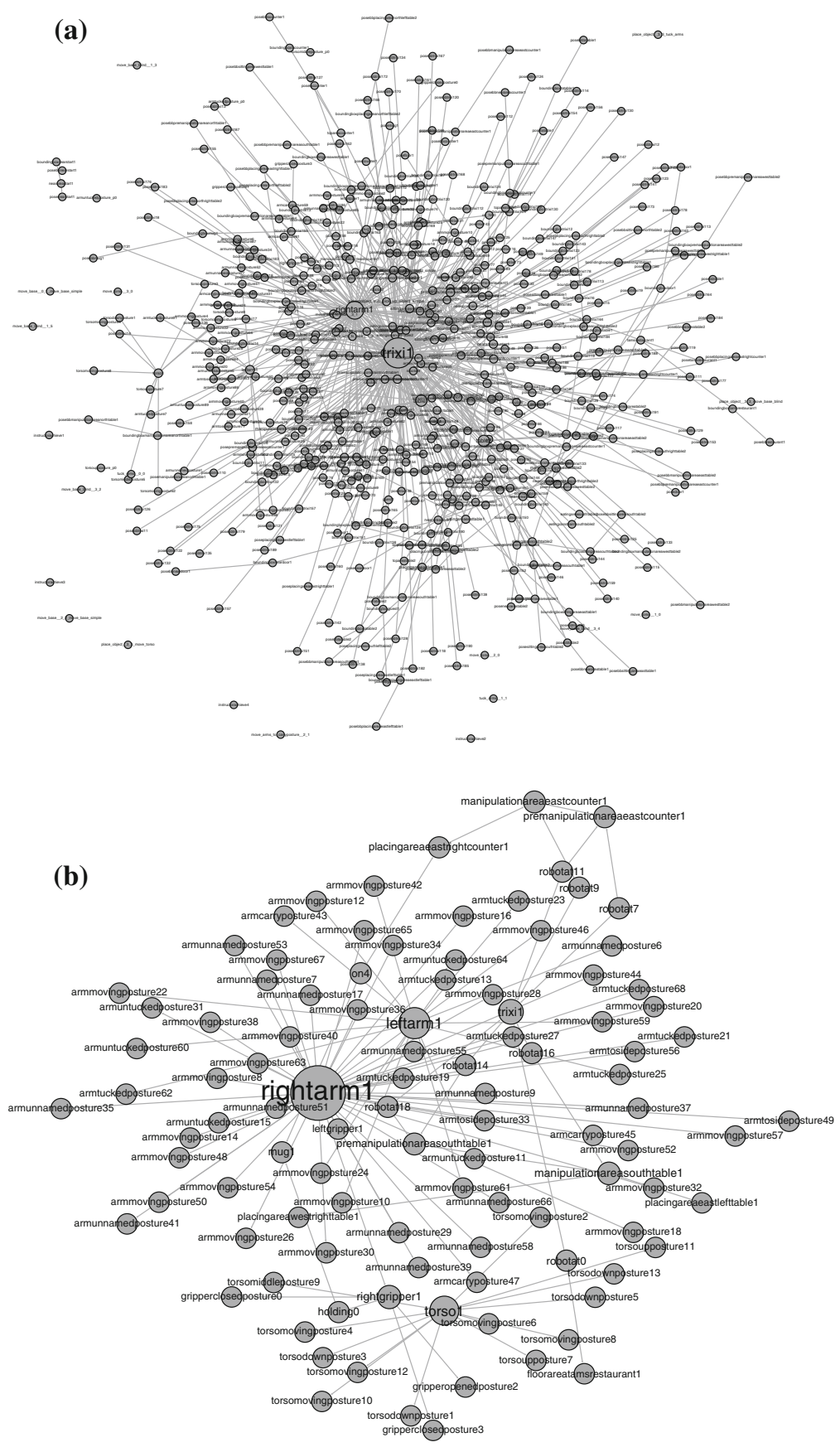

Fig. 2 Visualizing serve a coffee experience in Yifan Hu Multilevel layout. a Before filtering (a total of 649 uents), b after filtering (a total of 118 uents) 


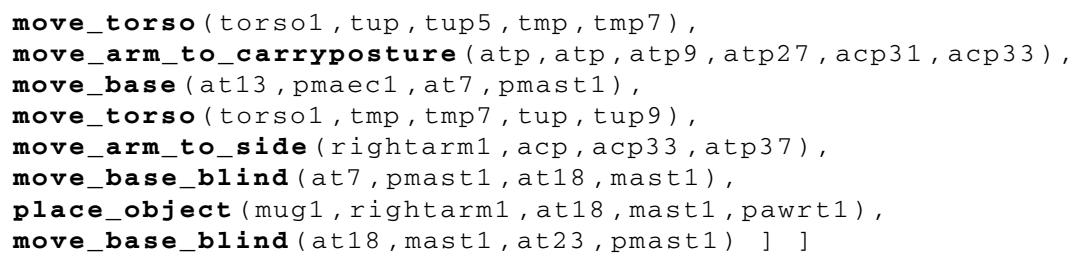

Listing 4 Representation of an experience of serving a coffee to a guest.

The proposed conceptualization approach is a combination of deductive generalization with different forms of abstraction. Deductive generalization (similar to explanation-based learning or EBL) [20] replaces constants in a real experience by variables in a way that is consistent with the known pre- and postconditions of actions. Each constant, wherever it appears playing the same role in the concrete experience, is consistently replaced by the same variable.

Applying abstraction is a common approach to reduce the level of details in a representation, allowing it to be more easily adapted to new situations. One of the abstraction steps in this approach is based on taxonomy of operators. To achieve this, (1) different primitive operators are mapped onto the same abstract operator; (2) some primitive operators are mapped onto an empty operator, meaning they are not included in the learned schema; and (3) some arguments of operators are not included in their corresponding abstract operators.

Since abstraction drops the details, extracting some other features would help to capture the essence of the experience. These features are properties of objects involved in an experience. The key propositions (KeyProps) extracted from the semantic memory through ego network-based graph filtering are used for discovering potentially relevant features. For each argument in every abstract operator, all possible relations with the key entities (the arguments of taught task), GUEST in this example, are extracted within a two-step neighborhood. These features are eventually used in planning to guide the search process toward solving a given problem. Listing 5 shows the learned serve coffee schema after generalization, abstraction, and feature extraction.

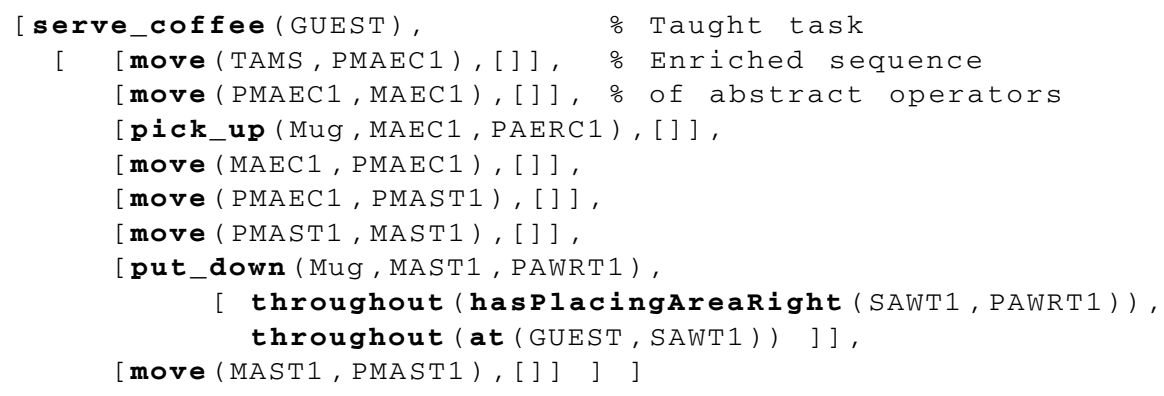

Listing 5 Serve coffee schema learned by conceptualizer.

The features hasPlacingAreaRight and at assert that PAWRT1 (Placing Area) belongs to SAWT1 (SittingArea) and the GUEST sits in SAWT1, respectively. This plan schema will be used as heuristic in planning to select, among multiple 
alternative solutions, the one that comes closer to the experience originally used for conceptualization. For example, although plans may be derived for serving on the left or on the right of the guest, the serve_coffee schema will lead to a preference in which the guest is served on the right side.

\section{Conclusion}

This paper proposed a method for supervised acquisition and conceptualization of plan-based robot activity experiences, in the RACE project. The paper presented the ontological concepts describing experiences and user instructions. A command-line interface was developed to facilitate experience gathering. Temporal segmentation heuristics were used to extract a subset of fluents as part of an experience in the interval that some activities took place. Since an experience contains numerous irrelevant occurrences, an ego network-based graph filtering approach was utilized to filter out such insignificant fluents. Task relevant fluents were selected as egos and the first order neighbors of egos were extracted to comprise an experience. A conceptualization was carried out based on deductive generalization and abstraction. As a result, a plan schema was constructed, which can guide a planner to search a solution in the similar situations. Conceptualizing activities based on multiple examples and utilizing plan schemata to guide search for a solution are considered as future works.

Acknowledgments We would like to thank the other RACE project partners for their efforts in the integration and the demonstrations, and especially to the Technical Aspects of Multimodal Systems (TAMS) group, University of Hamburg, for making the PR2 robot available do the project.

\section{References}

1. Mugan, J., Kuipers, B.: Autonomous learning of high-level states and actions in continuous environments. IEEE Transactions on Autonomous Mental Development (TAMD) 4(1) (2012) $70-86$

2. Kompella, V.R., Luciw, M., Stollenga, M., Pape, L., Schmidhuber, J.: Autonomous learning of abstractions using curiosity-driven modular incremental slow feature analysis. In: Development and Learning and Epigenetic Robotics (ICDL), 2012 IEEE International Conference on, IEEE (2012) $1-8$

3. Kirsch, A.: Robot learning language - integrating programming and learning for cognitive systems. Robotics and Autononomous Systems 57(9) (September 2009) 943-954

4. Pardowitz, M., Knoop, S., Dillmann, R., Zollner, R.D.: Incremental learning of tasks from user demonstrations, past experiences, and vocal comments. IEEE Transactions on Systems, Man, and Cybernetics, Part B 37(2) (2007) 322-332

5. Kirsch, A.: Integration of Programming and Learning in a Control Language for Autonomous Robots Performing Everyday Activities. PhD thesis, Technische Universitaet Muenchen (2008)

6. Ekvall, S., Kragic, D.: Learning task models from multiple human demonstrations. In: Robot and Human Interactive Communication, 2006. ROMAN 2006. The 15th IEEE International Symposium on, IEEE (2006) 358-363 
7. Ekvall, S., Kragic, D.: Robot learning from demonstration: A task-level planning approach. International Journal of Advanced Robotic Systems 5(3) (2008)

8. Nicolescu, M.N., Mataric, M.J.: Natural methods for robot task learning: Instructive demonstrations, generalization and practice. In: Proceedings of the second international joint conference on Autonomous agents and multiagent systems, ACM (2003) 241-248

9. Schou, C., Damgaard, J.S., Bogh, S., Madsen, O.: Human-robot interface for instructing industrial tasks using kinesthetic teaching. In: Robotics (ISR), 2013 44th International Symposium on, IEEE (2013) 1-6

10. Rockel, S., Neumann, B., Zhang, J., Dubba, K.S.R., Cohn, A.G., S̆. Konečný, Mansouri, M., Pecora, F., Saffiotti, A., Günther, M., Stock, S., Hertzberg, J., Tomé, A.M., Pinho, A.J., Lopes, L.S., von Riegen, S., Hotz, L.: An ontology-based multi-level robot architecture for learning from experiences. In: Designing Intelligent Robots: Reintegrating AI II, AAAI Spring Symposium, Stanford (USA) (2013)

11. S̆. Konečný, Stock, S., Pecora, F., Saffiotti, A.: Planning domain + execution semantics: a way towards robust execution? In: accepted to present in Qualitative Representations for Robots, AAAI Spring Symposium. (2014)

12. S. Stock, M.G., Hertzberg, J.: Generating and executing hierarchical mobile manipulation plans. In: ISR/Robotik 2014, Munich (accepted). (2014)

13. Tulving, E.: Episodic memory and autonoesis: Uniquely human? In H. S. Terrace,J.M., ed.: The Missing Link in Cognition. Oxford Univ. Press, NewYork, NY (2005) 4-56

14. Lim, G.H., Oliveira, M., Mokhtari Hassanabad, V., Kasaei, S.H., Seabra Lopes, L., Maria Tomé, A.: Interactive teaching and experience extraction for learning about objects and robot activities. In: RO-MAN 2014, IEEE (accepted). (2014)

15. Chauhan, A., Seabra Lopes, L., Tomé, A.M., Pinho, A.: Towards supervised acquisition of robot activity experiences: an ontology-based approach. In: 16th Portuguese Conference on Artificial Intelligence - EPIA'2013. (2013)

16. Lim, G.H., Suh, I.H., Suh, H.: Ontology-based unified robot knowledge for service robots in indoor environments. Systems, Man and Cybernetics, Part A: Systems and Humans, IEEE Transactions on 41(3) (2011) 492-509

17. Newman, M.E.: Ego-centered networks and the ripple effect. Social Networks 25(1) (2003) 83-95

18. Hu, Y.: Efficient, high-quality force-directed graph drawing. Mathematica Journal 10(1) (2005) 37-71

19. Seabra Lopes, L.: Failure recovery planning for robotized assembly based on learned semantic structures. In: IFAC Workshop on Intelligent Assembly and Disassembly (IAD'2007). (2007) 65-70

20. Levine, G., DeJong, G.: Explanation-based acquisition of planning operators. In: ICAPS. (2006) $152-161$ 